\title{
Liouville-type results in two dimensions for stationary points of functionals with linear growth
}

\author{
Michael Bildhauer and Martin Fuchs
}

\begin{abstract}
We consider elliptic systems generated by variational integrals of linear growth satisfying the condition of $\mu$-ellipticity for some exponent $\mu>1$ and prove that stationary points $u: \mathbb{R}^{2} \rightarrow \mathbb{R}^{N}$ with the property

$$
\limsup _{|x| \rightarrow \infty} \frac{|u(x)|}{|x|}<\infty
$$

must be affine functions. The latter condition can be dropped in the scalar case together with appropriate assumptions on the energy density providing an extension of Bernstein's theorem.

\section{Kaksiulotteisia Liouvillen-tyyppisiä tuloksia lineaarisesti kasvavien funktionaalien stationaarisille pisteille}

Tiivistelmä. Tarkastelemme lineaarisesti kasvavien variaatiointegraalien tuottamia yhtälösysteemeitä, jotka toteuttavat $\mu$-elliptisyysehdon jollakin eksponentilla $\mu>1$, ja todistamme, että kaikki ehdon

$$
\limsup _{|x| \rightarrow \infty} \frac{|u(x)|}{|x|}<\infty
$$

toteuttavat ratkaisut $u: \mathbb{R}^{2} \rightarrow \mathbb{R}^{N}$ ovat affiineja. Viimeksi mainittu ehto voidaan skalaaritapauksessa korvata sopivilla oletuksilla energiatiheydestä, mikä antaa yleistyksen Bernsteinin lauseelle.
\end{abstract}

\section{Introduction}

In this note we mainly present results of Liouville-type for entire solutions $u: \mathbb{R}^{2} \rightarrow$ $\mathbb{R}^{N}$ of the system

$$
\operatorname{div}[\nabla F(\nabla u)]=0 \quad \text { on } \mathbb{R}^{2},
$$

concentrating on the case of energy densities $F: \mathbb{R}^{2 N} \rightarrow \mathbb{R}$ with linear growth.

To be precise we assume that $F$ is of class $C^{2}\left(\mathbb{R}^{2 N}\right)$ satisfying with constants $M$, $\lambda, \Lambda>0$ and for some exponent $\mu>1$

$$
\begin{aligned}
|\nabla F(Z)| & \leq M \\
\lambda(1+|Z|)^{-\mu}|Y|^{2} & \leq D^{2} F(Z)(Y, Y) \leq \Lambda(1+|Z|)^{-1}|Y|^{2}
\end{aligned}
$$

for all $Y, Z \in \mathbb{R}^{2 N}$, where the first inequality in (1.3) expresses the fact that $F$ is a $\mu$-elliptic integrand. Note that (1.2) and (1.3) exactly correspond to the requirements of Assumption 4.1 in [2] and as outlined in Remark 4.2 of this reference, conditions (1.2) and (1.3) imply that $F$ is of linear growth in the sense that

$$
a|Z|-b \leq F(Z) \leq A|Z|+B, \quad Z \in \mathbb{R}^{2 N},
$$

holds with constants $a, A>0, B, b \geq 0$.

https://doi.org/10.54330/afm.114681

2020 Mathematics Subject Classification: Primary 49J40, 35J50.

Key words: Variational problems, linear growth, entire solutions in 2D, Liouville and Bernsteintype results.

(c) 2022 The Finnish Mathematical Society 
Note also that the "minimal surface case" is included by letting $F(Z):=(1+$ $\left.|Z|^{2}\right)^{1 / 2}$. In this case we have the validity of (1.3) with the choice $\mu=3$, and two families of densities satisfying (1.2) and (1.3) with prescribed exponent $\mu>1$ are given by

$$
F(Z):=\left\{\begin{array}{l}
\int_{0}^{|Z|} \int_{0}^{s}(1+r)^{-\mu} \mathrm{d} r \mathrm{~d} s \\
\int_{0}^{|Z|} \int_{0}^{s}\left(1+r^{2}\right)^{-\mu / 2} \mathrm{~d} r \mathrm{~d} s
\end{array}\right\}, \quad Z \in \mathbb{R}^{2 N} .
$$

Our results on the behaviour of global solutions of the Euler equations (1.1) with $\mu$-elliptic densities $F$ are as follows.

Theorem 1.1. Let $u \in C^{2}\left(\mathbb{R}^{2}, \mathbb{R}^{N}\right)$ denote a solution of (1.1) with density $F$ such that (1.2) and (1.3) hold.

a) Suppose that in addition

$$
\lim _{|x| \rightarrow \infty} \frac{|u(x)|}{|x|}=0 .
$$

Then $u$ is a constant function.

b) If the function $u$ has the property

$$
\sup _{x \in \mathbb{R}^{2}}|\nabla u(x)|<\infty
$$

then $u$ is affine.

c) If we have

$$
\limsup _{|x| \rightarrow \infty} \frac{|u(x)|}{|x|}<\infty,
$$

then the conclusion of $b$ ) holds.

Remark 1.1. a) Clearly (1.4) holds in the case that $u$ is a bounded solution, and evidently (1.5) implies (1.6).

b) We do not know if there are versions of Theorem 1.1 for entire solutions $u: \mathbb{R}^{n} \rightarrow \mathbb{R}^{N}$ of (1.1) in the case $n \geq 3$.

c) Our discussion of smooth solutions of the system (1.1) includes the vector case $N>1$ for densities $F$ of linear growth. The existence of smooth solutions is known provided that $\mu$ is not too large and provided that $F(Z)=f(|Z|)$. It is a challenging question whether the smoothness of solutions remains true (to some extend) if the second hypothesis is dropped.

Before presenting the proof of Theorem 1.1 we wish to mention that there exists a variety of Liouville-type theorems for entire solutions $u: \mathbb{R}^{n} \rightarrow \mathbb{R}^{N}, n \geq 2, N \geq 1$, of systems of the form (1.1) (and even for nonhomogeneous systems not generated by a density $F$ ) assuming that $F$ is of superlinear growth. The interested reader should consult the references on this topic quoted for example in the textbooks [12], [13], [14], [16], [22] and [25]. A nice survey is also presented in [6].

Besides this more general discussion the validity of Liouville theorems for harmonic maps between Riemannian manifolds turned out to be a useful tool for the analysis of the geometric properties of the underlying manifolds. Without being complete we refer to [4], [5], [17], [18], [19], [20], [27] and [28].

Liouville theorems are also of interest in the setting of fluid mechanics, where in the stationary case (1.1) is replaced by a nonlinear variant of the Navier-Stokes equation with dissipative potential $F$ of superlinear growth and the incompressibility 
condition $\operatorname{div} u=0$ for the velocity field $u: \mathbb{R}^{n} \rightarrow \mathbb{R}^{n}$ has to be added. The validity of Liouville theorems has been established in the 2-D-case, i.e. for $n=2$, for instance in the papers [3], [8], [9], [10], [11], [15], [21], [23], [29] and [30]. We like to mention that the case of potentials $F$ satisfying (1.2) and (1.3) is treated in [10] assuming $\mu<2$.

As it stands, the conclusions of Theorem $1.1 \mathrm{~b}$ ) and c) are in the spirit of Bernstein's theorem (see [1]) for nonparametric minimal surfaces, where in this particular setting conditions like (1.5) or (1.6) are seen to be superfluous. For completeness we specialize the Bernstein result obtained by Farina, Sciunzi and Valdinoci in Theorem 1.4 of their paper [7] to the case of linear growth integrands.

Theorem 1.2. Consider a function $g \in C^{2}([0, \infty))$ such that with constants $a_{1}$, $a_{3}, a_{5}>0, a_{2}, a_{4} \geq 0$ we have for some exponent $\mu \geq 1$

$$
\begin{gathered}
g^{\prime}(0)=0, \quad g^{\prime \prime}(t)>0 \quad \text { for } t>0, \\
a_{1} t-a_{2} \leq g(t) \leq a_{3} t+a_{4} \quad \text { for } t \geq 0, \\
g^{\prime \prime}(t) \leq a_{5}(1+t)^{-\mu} \quad \text { for } t \geq 0 .
\end{gathered}
$$

Let $F: \mathbb{R}^{2} \rightarrow \mathbb{R}, F(Z):=g(|Z|)$, and consider a solution $u: \mathbb{R}^{2} \rightarrow \mathbb{R}$ of (1.1) being of class $C^{2}$. Then $u$ is an affine function provided that $\mu \geq 3$.

Remark 1.2. a) Note that the minimal surface case is included with the choices $g(t)=\sqrt{1+t^{2}}$ and $\mu=3$, moreover, we can cover the examples stated in front of Theorem 1.1 provided that $\mu \geq 3$.

b) To our knowledge it is an unsolved problem, if Theorem 1.2 remains true for exponents $\mu \in(1,3)$.

c) Roughly speaking it follows from the work [26] of J.C.C. and J. Nitsche that the Bernstein property fails for the equation

$$
0=\operatorname{div}\left[\frac{g^{\prime}(|\nabla u|)}{|\nabla u|} \nabla u\right]
$$

if the density of $g$ is elliptic and of superlinear growth including even the nearly linear case $g(t)=t \ln (1+t)$, i.e. there exist non-affine solutions $u: \mathbb{R}^{2} \rightarrow \mathbb{R}$. However, the Nitsche criterion does not apply to integrands of linear growth as considered in Theorem 1.2 (see Remark 4.1).

d) From the identity

$$
D^{2} F(Z)(X, X)=\frac{1}{|Z|} g^{\prime}(|Z|)\left[|X|^{2}-\frac{1}{|Z|^{2}}(X \cdot Z)^{2}\right]+g^{\prime \prime}(|Z|) \frac{1}{|Z|^{2}}(X \cdot Z)^{2},
$$

$X, Z \in \mathbb{R}^{2}$, it follows that (observing the boundedness of $g^{\prime}$ )

$$
\min \left\{g^{\prime \prime}(|Z|), \frac{g^{\prime}(|Z|)}{|Z|}\right\}|X|^{2} \leq D^{2} F(Z)(X, X) \leq \Lambda(1+|Z|)^{-1}|X|^{2},
$$

i.e. the second inequality in (1.3) holds with some constant $\Lambda>0$. For $t \geq 1$ we have the lower bound $g^{\prime}(t) / t \geq c / t$, which by (1.9) means that in fact $g^{\prime \prime}(|Z|)$ measures the degree of ellipticity of $D^{2} F(Z)$. This shows that the integrand $F$ in general is not $\mu$-elliptic in the sense of the first inequality from (1.3): according to (1.9) the power $t^{-\mu}$ just acts as an upper bound for the values $g^{\prime \prime}(t)$. Thus we have the "Bernstein property" for any density $F(Z)=g(|Z|)$ of linear growth and for which $g^{\prime \prime}(t)=O\left(t^{-3}\right)$ as $t \rightarrow \infty$. 


\section{Proof of Theorem 1.1, Part a)}

In the weak formulation of (1.1), i.e. in the equation

$$
\int_{\mathbb{R}^{2}} \nabla F(\nabla u): \nabla \varphi \mathrm{d} x=0, \quad \varphi \in C_{0}^{1}\left(\mathbb{R}^{2}, \mathbb{R}^{N}\right),
$$

the function $\varphi$ is replaced by $\partial_{\alpha} \varphi\left(\alpha \in\{1,2\}\right.$ fixed), where now $\varphi \in C_{0}^{2}\left(\mathbb{R}^{2}, \mathbb{R}^{N}\right)$ is assumed. With an integration by parts we obtain from (2.1)

$$
\int_{\mathbb{R}^{2}} D^{2} F(\nabla u)\left(\partial_{\alpha} \nabla u, \nabla \varphi\right) \mathrm{d} x=0
$$

Note that by approximation equation (2.2) extends to $\varphi \in C_{0}^{1}\left(\mathbb{R}^{2}, \mathbb{R}^{N}\right)$, thus we may choose $\varphi=\eta^{2} \partial_{\alpha} u \in C_{0}^{1}\left(\mathbb{R}^{2}, \mathbb{R}^{N}\right)$ in $(2.2)$, where $\eta \in C_{0}^{1}\left(\mathbb{R}^{2}\right)$, spt $\eta \subset B_{2 R}(0)$, $\eta \equiv 1$ on $B_{R}(0), 0 \leq \eta \leq 1,|\nabla \eta| \leq c R^{-1}$. Then by Cauchy-Schwarz's and Young's inequality we have (summation w.r.t. $\alpha=1,2$ )

$$
\begin{aligned}
& \int_{B_{2 R}(0)} \eta^{2} D^{2} F(\nabla u)\left(\partial_{\alpha} \nabla u, \partial_{\alpha} \nabla u\right) \mathrm{d} x \\
& \leq c \int_{B_{2 R}(0)} D^{2} F(\nabla u)\left(\nabla \eta \otimes \partial_{\alpha} u, \nabla \eta \otimes \partial_{\alpha} u\right) \mathrm{d} x .
\end{aligned}
$$

The hypotheses (1.2) and (1.3) yield

$$
\begin{aligned}
\int_{B_{R}(0)}(1+|\nabla u|)^{-\mu}\left|\nabla^{2} u\right|^{2} \mathrm{~d} x & \leq c R^{-2} \int_{B_{2 R}(0)-B_{R}(0)} \frac{|\nabla u|^{2}}{\sqrt{1+|\nabla u|^{2}}} \mathrm{~d} x \\
& \leq c R^{-2} \int_{B_{2 R}(0)-B_{R}(0)}|\nabla u| \mathrm{d} x
\end{aligned}
$$

and using the auxiliary inequality (2.9) of Lemma 2.1 given below we obtain for any $\varepsilon>0$

$$
\begin{aligned}
& \int_{B_{R}(0)}(1+|\nabla u|)^{-\mu}\left|\nabla^{2} u\right|^{2} \mathrm{~d} x \\
& \leq \frac{c}{R^{2}} \int_{B_{2 R}(0)-B_{R}(0)}[\varepsilon+c(\varepsilon)(\nabla F(\nabla u)-\nabla F(0)): \nabla u] \mathrm{d} x .
\end{aligned}
$$

With (2.1) we also have

$$
\int_{\mathbb{R}^{2}}(\nabla F(\nabla u)-\nabla F(0)): \nabla \varphi \mathrm{d} x=0, \quad \varphi \in C_{0}^{1}\left(\mathbb{R}^{2}, \mathbb{R}^{N}\right),
$$

where we now choose $\varphi=\tilde{\eta}^{2} u, \tilde{\eta} \in C_{0}^{1}\left(\mathbb{R}^{2}\right), \tilde{\eta} \equiv 1$ on $B_{2 R}(0)-B_{R}(0)$, spt $\eta \subset$ $B_{5 R / 2}(0)-\bar{B}_{R / 2}(0), 0 \leq \tilde{\eta} \leq 1,|\nabla \tilde{\eta}| \leq c / R$.

With this choice (2.6) gives

$$
\begin{aligned}
\int_{\mathbb{R}^{2}}(\nabla F(\nabla u)-\nabla F(0)): \nabla u \tilde{\eta}^{2} \mathrm{~d} x & =-2 \int_{\mathbb{R}^{2}} \tilde{\eta}(\nabla F(\nabla u)-\nabla F(0)):(\nabla \tilde{\eta} \otimes u) \mathrm{d} x \\
& \leq c R^{-1} \int_{B_{5 R / 2}(0)-B_{R / 2}(0)}|u| \mathrm{d} x \\
& \leq c R \sup _{B_{5 R / 2}(0)-\bar{B}_{R / 2}(0)}|u|,
\end{aligned}
$$

where our assumption (1.2) is used. 
By the definition of $\tilde{\eta}$ we obtain using (2.7)

$$
\begin{aligned}
\int_{B_{2 R}(0)-B_{R}(0)}(\nabla F(\nabla u)-\nabla F(0)): \nabla u \mathrm{~d} x & \leq \int_{\mathbb{R}^{2}}(\nabla F(\nabla u)-\nabla F(0)): \nabla u \tilde{\eta}^{2} \mathrm{~d} x \\
& \leq c R \sup _{B_{5 R / 2}(0)-\bar{B}_{R / 2}(0)}|u| .
\end{aligned}
$$

If we insert (2.8) into inequality (2.5) and pass to the limit $R \rightarrow \infty$ recalling (1.4), we obtain for any $\varepsilon>0$

$$
\int_{\mathbb{R}^{2}}(1+|\nabla u|)^{-\mu}\left|\nabla^{2} u\right|^{2} \mathrm{~d} x \leq c \varepsilon
$$

hence $\nabla^{2} u \equiv 0$ and therefore we find $A \in \mathbb{R}^{2 N}, a \in \mathbb{R}^{N}$ such that

$$
u(x)=A x+a .
$$

Again we apply of the growth condition (1.4) and obtain $A=0$, hence the first part of Theorem 1.1 is established.

During the proof we made use of the elementary lemma

Lemma 2.1. Let $F \in C^{2}\left(\mathbb{R}^{2 N}\right)$ just satisfy the first inequality of (1.3) and let

$$
\theta(r):=\frac{\lambda}{\mu-1}\left[1-(1+r)^{1-\mu}\right], \quad r \geq 0 .
$$

Then it holds for any $\varepsilon>0$ and all $Z \in \mathbb{R}^{2 N}$

$$
|Z| \leq \varepsilon+\theta^{-1}(\varepsilon)[\nabla F(Z)-\nabla F(0)]: Z \text {. }
$$

Proof of Lemma 2.1. We fix $\varepsilon>0$. If $|Z| \geq \varepsilon$ then

$$
|Z| \theta(|Z|) \geq|Z| \theta(\varepsilon),
$$

which implies

$$
|Z| \leq \theta^{-1}(\varepsilon)|Z| \theta(|Z|),
$$

and if $Z \in \mathbb{R}^{2 N}$ is arbitrarily given, we have

$$
|Z| \leq \varepsilon+\theta^{-1}(\varepsilon)|Z| \theta(|Z|) .
$$

Moreover,

$$
\theta(|Z|)|Z| \leq[\nabla F(Z)-\nabla F(0)]: Z
$$

easily follows from

$$
[\nabla F(Z)-\nabla F(0)]: Z=\int_{0}^{1} \frac{\mathrm{d}}{\mathrm{d} t} \nabla F(t Z): Z \mathrm{~d} t=\int_{0}^{1} D^{2} F(t Z)(Z, Z) \mathrm{d} t
$$

and the first inequality in (1.3) as outlined in [2], formula (1), p. 98, and (2.10) gives (2.9).

Remark 2.1. Clearly Lemma 2.1 is not limited to the case $n=2$ and without condition (1.3) it would be sufficient to assume (2.10) for an increasing non-negative function $\theta:[0, \infty) \rightarrow \mathbb{R}$. 


\section{Proof of Theorem 1.1, Parts b) and c)}

For Part b) we remark, that the idea of applying a Liouville argument to the derivatives of solutions, which are seen to solve an appropriate elliptic equation, has been successfully used by Moser [24], Theorem 6, with the result that entire solutions of the minimal surface equation with bounded gradients in fact must be affine functions in any dimension $n \geq 2$.

In our setting, i.e. for $n=2$ together with $N \geq 1$, one may just follow the arguments presented in [12], Chapter III, p. 82, for an elementary proof essentially based on the "hole-filling" technique.

In Theorem 1.1, Part b) turns out to be a corollary of Part c), which we now prove following some ideas given in [11].

As in the proof of the first part of Theorem 1.1 we obtain from (2.3) the following variant of inequality $(2.4)$

$$
\int_{B_{R}(0)} D^{2} F(\nabla u)\left(\partial_{\alpha} \nabla u, \partial_{\alpha} \nabla u\right) \mathrm{d} x \leq c R^{-2} \int_{B_{2 R}(0)-B_{R}(0)}|\nabla u| \mathrm{d} x
$$

and, as outlined after (2.4), (3.1) gives for all $R>0$ and with the choice $\varepsilon=1$

$$
\int_{B_{R}(0)} D^{2} F(\nabla u)\left(\partial_{\alpha} \nabla u, \partial_{\alpha} \nabla u\right) \mathrm{d} x \leq c\left[1+R^{-1} \sup _{B_{5 R / 2}(0)-B_{R / 2}(0)}|u|\right] .
$$

Inequality (3.2) shows, using (1.6),

$$
\int_{\mathbb{R}^{2}} D^{2} F(\nabla u)\left(\partial_{\alpha} \nabla u, \partial_{\alpha} \nabla u\right) \mathrm{d} x<\infty .
$$

We finally claim that

$$
\int_{\mathbb{R}^{2}} D^{2} F(\nabla u)\left(\partial_{\alpha} \nabla u, \partial_{\alpha} \nabla u\right) \mathrm{d} x=0,
$$

which gives $\left|\nabla^{2} u\right|=0$, hence the proof will be complete.

To prove (3.4) we again consider (2.2) and choose $\varphi$ as done after this inequality. We obtain with $T_{R}:=B_{2 R}(0)-\bar{B}_{R / 2}(0)$ using the Cauchy-Schwarz inequality

$$
\begin{aligned}
\int_{\mathbb{R}^{2}} D^{2} F(\nabla u)\left(\partial_{\alpha} \nabla u, \partial_{\alpha} \nabla u\right) \eta^{2} \mathrm{~d} x= & -2 \int_{T_{R}} D^{2} F(\nabla u)\left(\eta \partial_{\alpha} \nabla u, \nabla \eta \otimes \partial_{\alpha} u\right) \mathrm{d} x \\
\leq & c\left[\int_{T_{R}} \eta^{2} D^{2} F(\nabla u)\left(\partial_{\alpha} \nabla u, \partial_{\alpha} \nabla u\right) \mathrm{d} x\right]^{\frac{1}{2}} \\
& \cdot\left[\int_{T_{R}} D^{2} F(\nabla u)\left(\nabla \eta \otimes \partial_{\alpha} u, \nabla \eta \otimes \partial_{\alpha} u\right) \mathrm{d} x\right]^{\frac{1}{2}} \\
= & : I_{1}(R) \cdot I_{2}(R) .
\end{aligned}
$$

We recall (3.3) which gives

$$
I_{1}(R) \rightarrow 0 \quad \text { as } R \rightarrow \infty .
$$

Assumption (1.3) yields the estimate

$$
I_{2}(R) \leq c\left[R^{-2} \int_{T_{R}}|\nabla u| \mathrm{d} x\right]^{\frac{1}{2}} .
$$


Thus we obtain (3.4), if we can prove

$$
\int_{B_{R}(0)}|\nabla u| \mathrm{d} x \leq c\left(1+R^{2}\right) .
$$

For (3.5) we use $(2.9)$ (recall $\eta \equiv 1$ on $B_{R}(0)$ ) with the choice $\varepsilon=1$, hence (compare the derivation of $(2.7))$

$$
\begin{aligned}
\int_{B_{R}(0)}|\nabla u| \mathrm{d} x & \leq\left|B_{R}(0)\right|+c \int_{B_{R}(0)}[\nabla F(\nabla u)-\nabla F(0)]: \nabla u \mathrm{~d} x \\
& \leq\left|B_{R}(0)\right|+c \int_{B_{2} R(0)} \eta^{2}[\nabla F(\nabla u)-\nabla F(0)]: \nabla u \mathrm{~d} x \\
& \leq c\left[R^{2}+R \sup _{T_{R}}|u|\right] \\
& =c R^{2}\left[1+\frac{1}{R} \sup _{T_{R}}|u|\right],
\end{aligned}
$$

and our hypothesis (1.6) gives (3.4), hence the proof of Theorem 1.1 is complete.

\section{Proof of Theorem 1.2}

We follow the lines of [7] by checking the hypotheses of Theorem 1.4 in this reference. We let

$$
a(t):=\frac{g^{\prime}(t)}{t}, \quad t>0
$$

and observe that on account of (1.7) the function $a$ continuously extends to $t=0$ by letting $a(0)=g^{\prime \prime}(0)$. Obviously $a$ satisfies (1.2) from [7] $\left(g^{\prime}\right.$ is strictly increasing and thereby positive on $(0, \infty)$ due to $(1.7))$, and since for any $t>0$ it holds (compare

$$
\lambda_{1}(t):=a(t)+t a^{\prime}(t)=g^{\prime \prime}(t)
$$

we get (1.3) in [7]. At the same time assumption (A2) from [7] is obvious by formula (4.1) and our requirements concerning $g$. Moreover, the stability condition (see (1.11) in [7]) follows from

$$
\int_{\mathbb{R}^{2}}(A(\nabla u) \nabla \phi) \cdot \nabla \phi \mathrm{d} x \geq 0
$$

for any $\phi \in C_{0}^{1}\left(\mathbb{R}^{2}\right)$ with matrix $\left(Z \in \mathbb{R}^{2}-\{0\}\right)$

$$
A_{i j}(Z):=|Z|^{-1} a^{\prime}(|Z|) Z_{i} Z_{j}+a(|Z|) \delta_{i j}
$$

by observing that (recall $(4.1),(1.7)$ )

$$
\begin{aligned}
(A(Y) Z) \cdot Z & =|Y|^{-1}\left[g^{\prime \prime}(|Y|)|Y|^{-1}-g^{\prime}(|Y|)|Y|^{-2}\right](Y \cdot Z)^{2}+|Y|^{-1} g^{\prime}(|Y|)|Z|^{2} \\
& \geq g^{\prime}(|Y|)\left[|Y|^{-1}|Z|^{2}-|Y|^{-3}(Y \cdot Z)^{2}\right] \geq 0
\end{aligned}
$$

on account of $g^{\prime}(|Y|)>0$ for $Y \neq 0$.

It remains to check (1.17) and (1.18) in [7]: since $g$ is convex (see (1.7)) and of linear growth (compare (1.8)) the boundedness of $g^{\prime}$ follows, hence we get (1.17) and by monotonicity $g_{\infty}^{\prime}:=\lim _{t \rightarrow \infty} g^{\prime}(t)$ exists in $(0, \infty)$. By $(4.2)$ the function $\lambda_{1}$ defined in (4.2) (see (1.5) of [7]) is just $g^{\prime \prime}$ so that (1.18) is a consequence of (1.9) and the aforementioned limit behaviour of $g^{\prime}$ provided we assume $\mu \geq 3$. From Theorem 1.4 
in [7] it follows that $u(x)=\tilde{u}(\omega \cdot x)$ for some $\omega \in \mathbb{R}^{2},|\omega|=1$, and a function $\tilde{u}$ : $\mathbb{R} \rightarrow \mathbb{R}$. Assuming $\omega=(1,0)$ (w.l.o.g.) we see that (1.1) implies

$$
\frac{\mathrm{d}}{\mathrm{d} t}\left[\frac{1}{\left|\tilde{u}^{\prime}\right|} g^{\prime}\left(\left|\tilde{u}^{\prime}\right|\right) \tilde{u}^{\prime}\right]=0
$$

hence

$$
\left|\tilde{u}^{\prime}\right|^{-1} g^{\prime}\left(\left|\tilde{u}^{\prime}\right|\right) \tilde{u}^{\prime} \equiv c
$$

for some $c \in \mathbb{R}$.

Case $1, c=0$. Recalling $g^{\prime}>0$ on $(0, \infty)$ equation $(4.4)$ yields $\tilde{u}^{\prime} \equiv 0$ and we are done.

Case 2, $c \neq 0$. Then (4.4) shows $\tilde{u}^{\prime}(t) \neq 0$ for any $t \in \mathbb{R}$, thus $g^{\prime}\left(\left|\tilde{u}^{\prime}\right|\right) \equiv|c|$ and in conclusion

$$
\tilde{u}^{\prime}(t) \in\left\{-\left(g^{\prime}\right)^{-1}(|c|),\left(g^{\prime}\right)^{-1}(|c|)\right\}
$$

for any $t \in \mathbb{R}$. But this immediately implies the constancy of $\tilde{u}^{\prime}$ and our claim follows.

Remark 4.1. Let us end with a short remark on the failure of the Nitsche criterion (condition (4) in [26]) for densities $g$ with the properties (1.7) and (1.8) from Theorem 1.2. The boundedness of $g^{\prime}$ in particular shows that

$$
\int_{1}^{\infty} g^{\prime \prime}(t) \mathrm{d} t<\infty, \text { thus } \int_{1}^{\infty} \frac{1}{\sqrt{s}} g^{\prime \prime}(\sqrt{s}) \mathrm{d} s<\infty
$$

Let us introduce the functions (compare [26])

$$
f(t):=g(\sqrt{t}), \quad \lambda(t):=\frac{2 f^{\prime \prime}(t)}{f^{\prime}(t)} .
$$

Elementary calculations show

$$
\frac{1+t \lambda(t)}{2+t \lambda(t)} \cdot \frac{1}{t}=\frac{g^{\prime \prime}(\sqrt{t})}{\frac{1}{\sqrt{t}} g^{\prime}(\sqrt{t})+g^{\prime \prime}(\sqrt{t})} \cdot \frac{1}{t}=\frac{1}{t+\frac{\sqrt{t}}{g^{\prime \prime}(\sqrt{t})} g^{\prime}(\sqrt{t})}
$$

and for $t \geq 1$ we obtain by the monotonicity of $g^{\prime}$

Recalling (4.5) it follows

$$
\frac{1+t \lambda(t)}{2+t \lambda(t)} \frac{1}{t} \leq \frac{1}{g^{\prime}(1)} \frac{g^{\prime \prime}(\sqrt{t})}{\sqrt{t}} \text {. }
$$

$$
\int_{1}^{\infty} \frac{1+t \lambda(t)}{2+t \lambda(t)} \frac{\mathrm{d} t}{t}<\infty
$$

which means that the "Satz" on p. 295 of [26] does not apply to the linear growth case.

\section{References}

[1] Bernstein, S.: Über ein geometrisches Theorem und seine Anwendung auf die partiellen Differentialgleichungen vom elliptischen Typus. - Math. Z. 26:1, 1927, 551-558.

[2] Bildhauer, M.: Convex variational problems. Linear, nearly linear and anisotropic growth conditions. - Lecture Notes in Math. 1818, Springer, Berlin, 2003.

[3] Bildhauer, M., M. Fuchs, and G. Zhang: Liouville-type theorems for steady flows of degenerate power law fluids in the plane. - J. Math. Fluid Mech. 15:3, 2013, 583-616.

[4] Cheng, S. Y.: Liouville theorem for harmonic maps. - Proc. Sympos. Pure Math. XXXVI, Amer. Math. Soc., Providence, R.I., 1980, 147-151. 
[5] Eells, J., and L. Lemaire: Another report on harmonic maps. - Bull. London Math. Soc. $20: 5,1988,385-524$.

[6] FARINA, A.: Liouville-type theorems for elliptic problems. - In: Handbook of differential equations: stationary partial differential equations. Vol. IV, Elsevier/North-Holland, Amsterdam, 2007, 61-116.

[7] Farina, A., B. Sciunzi, and E. VAldinoci: Bernstein and De Giorgi type problems: new results via a geometric approach. - Ann. Sc. Norm. Super. Pisa Cl. Sci. (5) 7:4, 2008, 741-791.

[8] Fuchs, M.: Liouville theorems for stationary flows of shear thickening fluids in the plane. J. Math. Fluid Mech. 14:3, 2012, 421-444.

[9] Fuchs, M.: Variations on Liouville's theorem in the setting of stationary flows of generalized Newtonian fluids in the plane. - In: Proceedings of the St. Petersburg Mathematical Society. Vol. XV. Advances in mathematical analysis of partial differential equations 232, Amer. Math. Soc. Transl. Ser. 2, Providence, RI, 2014, 79-98.

[10] Fuchs, M., and J. MÜller: A Liouville theorem for stationary incompressible fluids of von Mises type. - Acta Math. Sci. Ser. B (Engl. Ed.) 39:1, 2019, 1-10.

[11] FuCHS, M., and G. ZHANG: Liouville theorems for entire local minimizers of energies defined on the class LlogL and for entire solutions of the stationary Prandtl-Eyring fluid model. - Calc. Var. Partial Differential Equations 44:1-2, 2012, 271-295.

[12] Giaquinta, M.: Multiple intergals in the calculus of variations and nonlinear ellipitc systems. - Ann. of Math. Stud. 105, Princeton Univ. Press, Princeton, NJ, 1983.

[13] Giaquinta, M., and L. Martinazzi: An introduction to the regularity theory for elliptic systems, harmonic maps and minimal graphs. - Lecture Notes. Scuola Normale Superiore di Pisa (New Series) 11, Edizioni della Normale Superiore di Pisa (Nuova Serie), Pisa, second edition, 2012.

[14] Gilbarg, D., and N. Trudinger: Elliptic partial differential equations of second order. Grundlehren Math. Wiss. 224, Springer, Berlin, Revised Third Printing, second edition, 1998.

[15] Gilbarg, D., and H.F. Weinberger: Asymptotic properties of steady plane solutions of the Navier-Stokes equations with bounded Dirichlet integral. - Ann. Scuola Norm. Sup. Pisa Cl. Sci. (4) 5:2, 1978, 381-404.

[16] Giusti, E.: Direct methods in the calculus of variations. - World Scientific Publishing Co., River Edge, NJ, 2003.

[17] Hildebrandt, S.: Liouville theorems for harmonic mappings, and an approach to Bernstein theorems. - Ann. of Math. Stud. 102, Seminar on Differential Geometry, Princeton University, Princeton, N.J., 1982, 107-131.

[18] Hildebrandt, S., J. Jost, and K. O. Widman: Harmonic mappings and minimal submanifolds. - Invent. Math. 62:2, 1980, 269-298.

[19] Hildebrandt, S., and H. Kaul: Two-dimensional variational problems with obstructions, and Plateau's problem for H-surfaces in a Riemannian manifold. - Comm. Pure Appl. Math. 25, 1972, 187-223.

[20] Hildebrandt, S., H. Kaul, and K. O. Widman: An existence theorem for harmonic mappings of Riemannian manifolds. - Acta Math. 138:1-2, 1977, 1-16.

[21] JIN, B. J., and K. KANG: Liouville theorem for the steady-state non-Newtonian Navier-Stokes equations in two dimensions. - J. Math. Fluid Mech. 16:2, 2014, 275-292.

[22] Jost, J.: Partial differential equations. - Grad. Texts in Math., Springer, New York, 2013.

[23] Koch, G., N. Nadirashvili, G. Seregin, and V. Šverák: Liouville theorems for the Navier-Stokes equations and applications. - Acta Math. 203:1, 2009, 83-105.

[24] Moser, J.: On Harnack's theorem for elliptic differential equations. - Comm. Pure Appl. Math. $14,1961,577-591$. 
[25] NeČAS, J.: Introduction to the theory of nonlinear elliptic equations. - Teubner-Texte zur Mathematik 52, Teubner Verlagsgesellschaft, Leipzig, 1983.

[26] Nitsche J., and J. Nitsche: Ein Kriterium für die Existenz nicht-linearer ganzer Lösungen elliptischer Differentialgleichungen. - Arch. Math. 10, 1959, 294-297.

[27] Schoen, R.: Analytic aspects of the harmonic map problem. - In: Seminar on nonlinear partial differential equations (Berkeley, Calif., 1983), Sci. Res. Inst. Publ. 2, Springer, New York, 1984, $321-358$.

[28] Schoen, R., and S. T. Yau: Harmonic maps and the topology of stable hypersurfaces and manifolds with non-negative Ricci curvature. - Comment. Math. Helv., 1976, 333-341.

[29] Zhang, G.: A note on Liouville theorem for stationary flows of shear thickening fluids in the plane. - J. Math. Fluid Mech. 15:4, 2013, 771-782.

[30] Zhang, G.: Liouville theorems for stationary flows of shear thickening fluids in 2D. Ann. Acad. Sci. Fenn. Math. 40:2, 2015, 889-905.

Received 4 January 2021 • Accepted 13 July 2021 • Published online 14 February 2022

Michael Bildhauer

Saarland University

Department of Mathematics

P.O. Box 151150

66041 Saarbrücken, Germany

bibi@math.uni-sb.de
Martin Fuchs

Saarland University

Department of Mathematics

P.O. Box 151150

66041 Saarbrücken, Germany

fuchs@math.uni-sb.de 